\section{Tocilizumab for polymyalgia rheumatica: a first or second line?}

To the Editor:

In the open-label uncontrolled trial recently published in the Annals of the Rheumatic Diseases, Devauchelle-Pensec et al ${ }^{1}$ showed high efficacy of tocilizumab as a first-line treatment for polymyalgia rheumatica (PMR). Twenty patients with PMR were treated with three tocilizumab intravenous infusions at a dose of $8 \mathrm{mg} / \mathrm{kg}$ with 4-week intervals followed by oral prednisone. At 12 weeks, the primary end-point of a PMR activity score $\leq 10$ was achieved in all patients; moreover, tocilizumab had impressive steroid-sparing effect (up to 70\%). Tocilizumab is a promising option for PMR and giant cell arteriitis (GCA). Its efficacy in the new-onset or relapsing GCA has recently been confirmed in the single-centre phase II randomised placebocontrolled study. ${ }^{2}$ Currently, tocilizumab is usually reserved for patients with recurrent PMR or GCA who do not respond to or poorly tolerate standard immunosuppression, though favourable results of the phase II study may justify its wider use for the induction of remission in the new-onset GCA. Should we use this biologic agent as a first-line therapy for PMR?

PMR has a relatively benign course and can be effectively treated with low-dose glucocorticoids. Apparently, glucocorticoid therapy can cause different side effects (eg, osteoporosis) but in lower doses its risk:benefit ratio is probably positive in the majority of patients with PMR, especially of younger age. In the study by Devauchelle-Pensec et al tocilizumab infusions were not associated with serious adverse events. Nevertheless, $14(70 \%)$ patients developed 22 drug-related reactions; the most common were leucopoenia, neutropenia and moderately severe infections. In four (25\%) patients, leucopoenia prompted a decrease in tocilizumab dosage or withholding of treatment. The same side effects were also frequent in patients with GCA who were treated with tocilizumab at a dose of $8 \mathrm{mg} / \mathrm{kg}^{2}$ Lowering the dosage of tocilizumab to $4 \mathrm{mg} / \mathrm{kg}$ that is widely used in rheumatoid arthritis may probably improve its safety in the elderly patients with PMR.

The response to tocilizumab was delayed. At 4 weeks, very low disease activity was achieved only in $45 \%$ of patients while in a recent prospective study complete response to glucocorticoids was found within 4 weeks in $71 \%$ of 125 patients with PMR. ${ }^{3}$ Notably, this difference was seen in different studies and may be confirmed only in head-to-head comparative trials.

Thus, efficacy of tocilizumab was achieved at the expense of relatively frequent and potentially dangerous side effects, delayed onset of activity and a high cost of biologic therapy. We agree with the authors that randomised controlled trials of tocilizumab in PMR are warranted though the results of the current study suggest that it is still more valuable as a secondline agent in patients with refractory or recurrent course of disease and/or toxicity of glucocorticoid. The candidates for initial tocilizumab monotherapy may include very old or frail patients with significant comorbidity in whom the risk of glucocorticoids side effects even at lower dosages can be unacceptable. The advantage of tocilizumab was its significant steroid-sparing effect, but the risk of infections and leucopoenia cannot be ignored and justifies careful monitoring. In our opinion, the combination of tocilizumab at a dose of $4 \mathrm{mg} / \mathrm{kg}$ with low-dose glucocorticoids may provide a more rapid effect with acceptable safety and deserves further studies.

\section{Sergey Moiseev, Pavel Novikov, Victor Fomin}

Clinic of Nephrology, Internal and Occupational Diseases, Sechenov First Moscow State Medical University, Moscow, Russia

Correspondence to Professor Sergey Moiseev, Clinic of Nephrology, Internal and Occupational Diseases, Sechenov First Moscow State Medical University, Rossolimo, 11/5, Moscow 119934, Russia; clinpharm@mtu-net.ru

Competing interests None declared.

Provenance and peer review Not commissioned; internally peer reviewed.

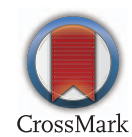

To cite Moiseev S, Novikov P, Fomin V. Ann Rheum Dis 2016;75:e47.

Accepted 23 March 2016

Published Online First 13 April 2016

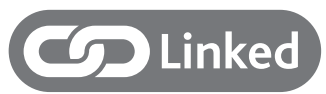

http://dx.doi.org/10.1136/annrheumdis-2016-209607

Ann Rheum Dis 2016;75:e47. doi:10.1136/annrheumdis-2016-209596

\section{REFERENCES}

1 Devauchelle-Pensec V, Berthelot JM, Cornec D, et al. Efficacy of first-line tocilizumab therapy in early polymyalgia rheumatica: a prospective longitudinal study. Ann Rheum Dis 2016;75:1506-10.

2 Villiger PM, Adler S, Kuchen S, et al. Tocilizumab for induction and maintenance of remission in giant cell arteritis: a phase 2, randomised, double-blind, placebocontrolled trial. Lancet 2016. Mar 4. pii: S0140-6736(16)00560-2. doi:10.1016/ S0140-6736(16)00560-2. [Epub ahead of print]

3 Dasgupta B, Cimmino MA, Maradit-Kremers H, et al. 2012 Provisional classification criteria for polymyalgia rheumatica: a European League Against Rheumatism/ American College of Rheumatology collaborative initiative. Ann Rheum Dis 2012;71:484-92. 